\title{
The efficacy and safety of continuous versus single-injection popliteal sciatic nerve block in outpatient foot and ankle surgery: a systematic review and meta- analysis
}

Hsuan-Hsiao Ma ${ }^{1,2}$, Te-Feng Arthur Chou ${ }^{1,2}$, Shang-Wen Tsai ${ }^{1,2^{*}}$, Cheng-Fong Chen ${ }^{1,2}$, Po-Kuei Wu ${ }^{1,2}$ and Wei-Ming Chen ${ }^{1,2}$

\begin{abstract}
Background: Continuous popliteal sciatic nerve block (CPSNB) has been performed in outpatient foot and ankle surgery as a regional anesthesia method to relieve postoperative pain. Its efficacy as well as safety is yet to be established. There are two purposes of this study: (1) to validate the efficacy of CPSNB with regards to better pain relief and reduced analgesics consumption; (2) to assess the safety of CPSNB.

Methods: We performed a comprehensive literature review on Web of Science, the Cochrane Library, PubMed and Embase and only included randomized controlled trials (RCTs). Five RCTs that compared the efficacy and safety of CPSNB with the single-injection popliteal sciatic nerve block group were included. The primary outcome parameters were visual analog scale (VAS) scores at postoperative 24,48 and $72 \mathrm{~h}$. The secondary outcome parameters were amount of oral analgesics consumed, overall patient satisfaction and need of admission after surgery. A sensitivity analysis was performed to explore the consistency of the results.

Results: In comparison with the single-injection group, CPSNB was associated with a lower VAS score at postoperative 24 and $48 \mathrm{~h}(p<0.05)$. There were no neuropathic symptoms or infection events after the nerve block. However, there were several minor complications associated with the pump and catheter system, with drug leakage being the most common complication ( $N=26$ of 187, 13.9\%).
\end{abstract}

Conclusion: CPSNB is an effective method in pain management for outpatient foot and ankle surgery. Both methods appear to be safe as none of the patients experienced neuropathic symptoms or infection. Further studies with larger sample size are needed to compare the risk of major complications between the two methods.

Level of evidence: l; meta-analysis.

Keywords: Complication, Continuous popliteal sciatic nerve block, Foot and ankle surgery, Outpatient surgery, Pain management

\footnotetext{
* Correspondence: ray366kimo@gmail.com

${ }^{1}$ Department of Orthopaedics and Traumatology, Taipei Veterans General

Hospital, No. 201, Sec 2, Shi-Pai Road, Taipei 112, Taiwan

${ }^{2}$ Department of Orthopaedics, School of Medicine, National Yang-Ming

University, Taipei, Taiwan
}

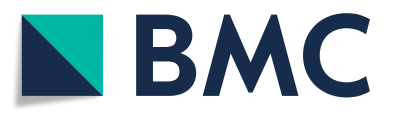

(c) The Author(s). 2019 Open Access This article is distributed under the terms of the Creative Commons Attribution 4.0 International License (http://creativecommons.org/licenses/by/4.0/), which permits unrestricted use, distribution, and reproduction in any medium, provided you give appropriate credit to the original author(s) and the source, provide a link to the Creative Commons license, and indicate if changes were made. The Creative Commons Public Domain Dedication waiver (http://creativecommons.org/publicdomain/zero/1.0/) applies to the data made available in this article, unless otherwise stated. 


\section{Introduction}

Foot and ankle surgery is increasingly performed in a same-day, outpatient setting but is associated with moderate to severe postoperative pain which may lead to prolonged post-anesthesia care unit (PACU) stay, unanticipated admission and decreased patient satisfaction [1-3]. Postoperative pain was one of the most common reasons for unanticipated admission, in which $57.6 \%$ of them were orthopaedic patients [4]. This moderate to severe postoperative pain following foot and ankle surgery can persist up to postoperative day 3 [5], which single-injection popliteal sciatic nerve block may not be adequate to manage the pain [6]. Therefore, continuous popliteal sciatic nerve block (CPSNB) has been developed as a potential pain management method. Several randomized controlled trials have been conducted to compare the efficacy and complications of CPSNB versus single-injection nerve block [7-11]. Some studies concluded that CPSNB was more effective in pain management than the single-injection nerve block [7, 9-11], while Elliot et al. suggested that it was still debatable whether the additional benefits of CPSNB are worthy of its extra time and cost involved because the pain scores were very low in both groups [8]. Therefore, the first aim of this meta-analysis was to determine the efficacy of CPSNB compared with single-injection group, as a postoperative pain management in patients who had undergone foot and ankle surgery.

In addition to the efficacy, safety concerns are another important issue that should be validated. Neuropathic symptoms and infection following the nerve block are two major complications mentioned in current literature
[12-16]. The incidence of neuropathic symptoms after CPSNB has been reported in some studies to be relatively low $[13,15]$, but recent studies found a higher rate [16-18]. Other complications including accidental falls, adverse drug reaction and other minor complications associated with the infusion system were also mentioned $[7-11,19]$. Thus the second aim of this meta-analysis was to assess the safety of CPSNB and to identify major and minor complications associated with CPSNB.

\section{Materials and methods}

\section{Search strategy}

We searched databases including Web of Science, the Cochrane Library, PubMed and Embase from the earliest record to December 2018, for randomized controlled trials (RCTs) that validated the efficacy and safety for CPSNB in outpatient foot and ankle surgery. We manually reviewed all the bibliographies of the included studies for relevent references. We excluded studies that were not written in English or not available in full text. Our search strategy included the following keywords in various combination: (popliteal block OR regional anesthesia OR nerve block OR popliteal sciatic nerve block) AND (ankle fracture OR calcaneal fracture OR foot and ankle surgery). We enrolled only RCTs and excluded comparative cohort studies, case series and case reports. The included studies should comprise at least two treatment arms: continuous popliteal sciatic nerve block and single-injection popliteal sciatic nerve block. The preferred reporting items for systematic reviews and meta-analysis (PRISMA) guidelines were applied in our search strategy (Fig. 1).

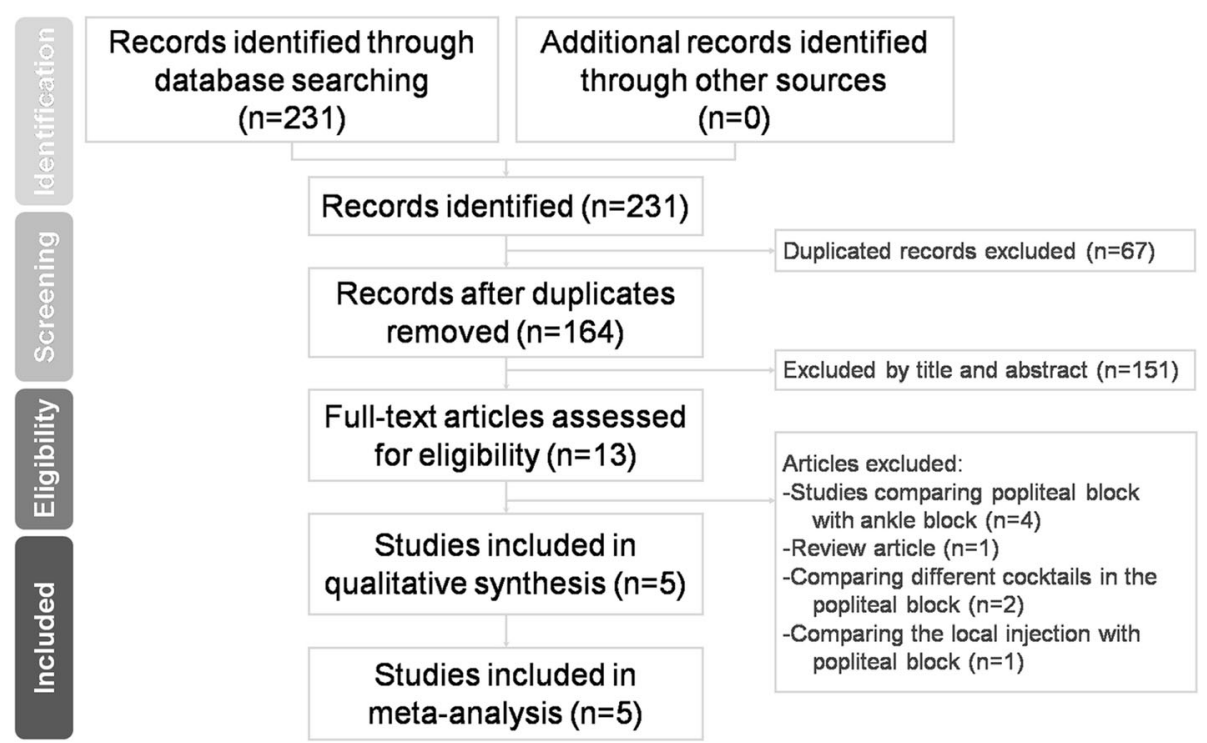

Fig. 1 Preferred reporting items for systematic reviews and meta-analysis (PRISMA) flow diagram for the searching and identification of included studies 


\section{Inclusion criteria and study selection}

All studies that were eligible for our analysis met the PICOS criteria (population, intervention, comparator, outcomes, study design). Population: patients who had undergone outpatient foot and ankle surgery. Intervention: Using continuous popliteal sciatic nerve block as the pain control method. Comparator: Single-injection popliteal sciatic nerve block. Outcomes: Visual analog scale (VAS) at different time points, amount of oral analgesics consumed, patient satisfaction and need of admission. Studies must have a follow-up rate of at least $80 \%$, and at least 1 of the above outcome parameters was included. Study design: interventional randomized controlled trials.

\section{Data extraction and quality assessment}

Two reviewers extracted data from all identified articles using a predetermined form. Information about the first author, year, study design, enrolled sample number, type of treatment arms, anesthesia method, approach of popliteal sciatic nerve block, regimen of infiltration analgesics, type of foot and ankle surgery, outcome parameters to evaluate pain, amount of oral analgesics consumed, patient satisfaction and need of admission after surgery were listed in Table 1. Jadad score was used to assess the quality of the included RCTs. The score ranged from 0 to 5 ; we defined a score of 4-5 points as good, 2-3 points as fair and $0-1$ point as a study with poor methodology. Discrepancies between the two reviewers were solved by discussion.

\section{Statistical analysis}

The standardized mean differences (SMDs) of postoperative VAS score at different time points between the CPSNB and single-injection popliteal sciatic nerve block was the primary outcome. The VAS scores from each study at 24, 48 and $72 \mathrm{~h}$ were included. A negative SMD value indicated CPSNB to be a favorable treatment option. The secondary outcomes were amount of oral analgesics consumed (assessed with SMD), overall patient satisfaction and need of admission after CPSNB or single-injection popliteal sciatic nerve that were assessed with odds ratio (OR). We used random effect model to pool individual SMDs and ORs. Analyses were performed using Comprehensive Meta-Analysis (CMA) software, version 3 (Biostat, Englewood, NJ, USA). Between-trial heterogeneity was determined by using $I^{2}$ tests; values $>50 \%$ were regarded as considerable heterogeneity. Statistical significance was defined as $p$-values $<0.05$. Due to concerns of inconsistency results in this study, the validity and robustness of effect estimates were adjusted accordingly. A sensitivity analyses was performed by excluding the one study which contained the smallest sample size of the pooled data. We used Trial Sequential Analysis software (Copenhagen Trial Unit, Copenhagen, Denmark) for the trial sequence analysis. Trial sequence analysis calculates required information size which compares well to a sample size calculation for a RCT according to an overall type I error of $5 \%$ and a power of $80 \%$ [20]. Two reviewers used Cochrane collaboration's tool for assessing risk of bias in each included randomized trials. Funnel plots and Egger's test were used to examine potential publication bias.

\section{Results}

A total of 231 relevant articles were identified according to our search strategy. Sixty-seven duplicate records were removed. One hundred and fifty-one studies were excluded by title and abstract. Based on the inclusion criteria, 8 studies were excluded after reading the full text. Finally, 5 articles that compared the efficacy of CPSNB in outpatient foot and ankle surgery with singleinjection popliteal sciatic nerve block were included for our meta-analysis. Baseline characteristics of the 5 included studies are summarized in Table 1. Quality of these five included RCTs were good (Jadad score 4-5 points).

\section{Meta-analysis results VAS score at 24, 48 and $72 \mathrm{~h}$}

The VAS score at 24,48 and $72 \mathrm{~h}$ were reported in 5 studies including a total of 208 patients. The analysis showed a significantly lower VAS score at $24 \mathrm{~h}$ in the CPSNB group in comparison with the single-injection group (SMD: -1.331; $95 \%$ CI -2.149 to -0.512 ; Heterogeneity: $\mathrm{I}^{2}=85.1$; Fig. 2). In addition, there was a lower VAS score at $48 \mathrm{~h}$ noted in the CPSNB group (SMD -1.034; 95\% CI - 1.755 to -0.314 ; Heterogeneity: $\mathrm{I}^{2}=82.2$; Fig. 3 ). Based on the trial sequence analysis, both methods had adequate sample size to compare the VAS scores at 24 and $48 \mathrm{~h}$ (Additional file 1: Figure S1 and Additional file 2: Figure S2). The VAS score at $72 \mathrm{~h}$ was not different between the groups (SMD: -0.491 ; 95\% CI - 1.016 to 0.033; Heterogeneity: $\mathrm{I}^{2}=69.7$; Fig. 4). However, this result was considered inconclusive because of inadequate number of studies according to the trial sequence analysis. (Additional file 3: Figure S3).

\section{Total amount of Oral analgesics consumed at $72 \mathrm{~h}$}

Total amount of oral analgesics consumed at $72 \mathrm{~h}$ was reported in 4 studies and included 158 patients. The pooled data from patients that received CPSNB was associated with less amount of oral analgesics consumed (SMD: -0.606 ; $95 \%$ CI -0.925 to -0.287 ; Heterogeneity: $\mathrm{I}^{2}=0$; Fig. 5). However, the enrolled number did not reach required information size. Thus, this result was considered inconclusive. (Additional file 4: Figure S4). 


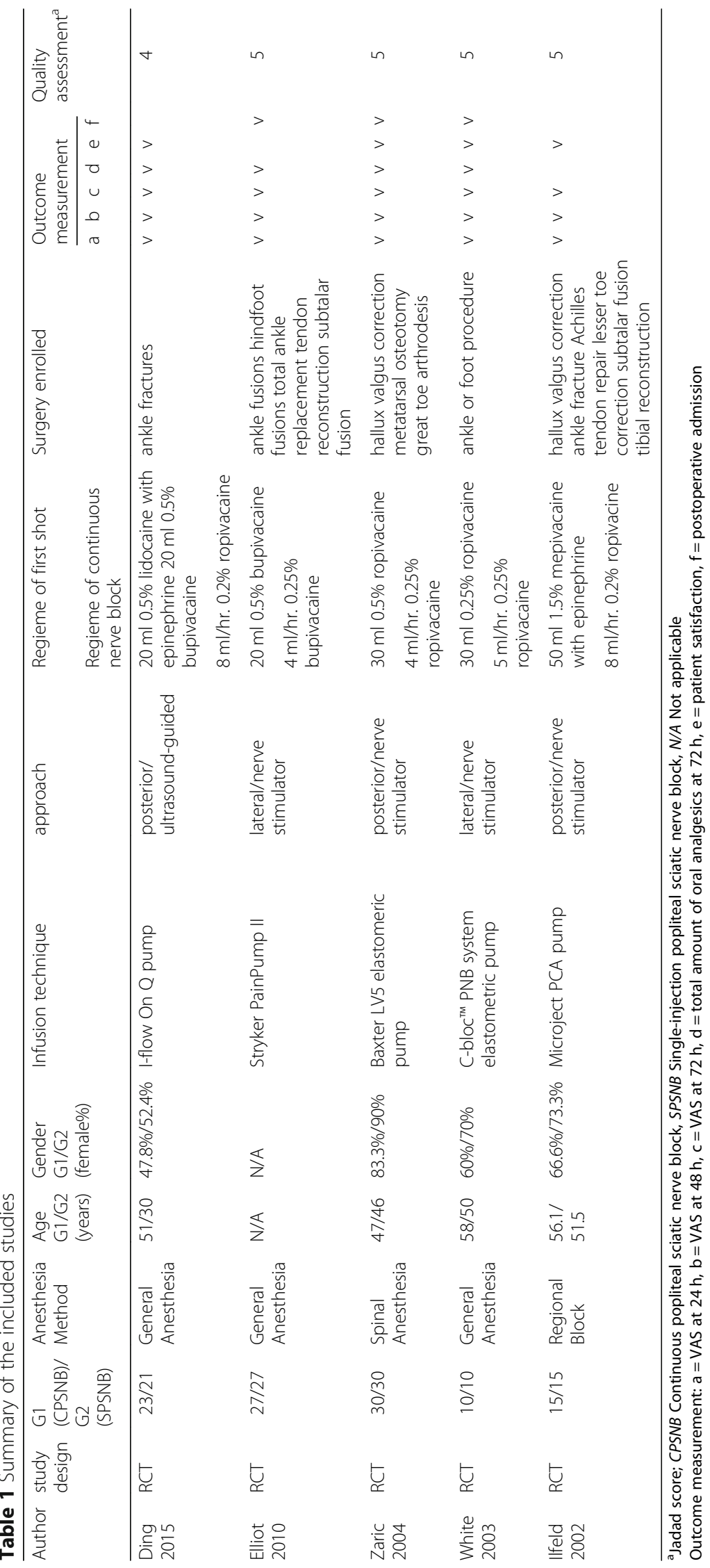




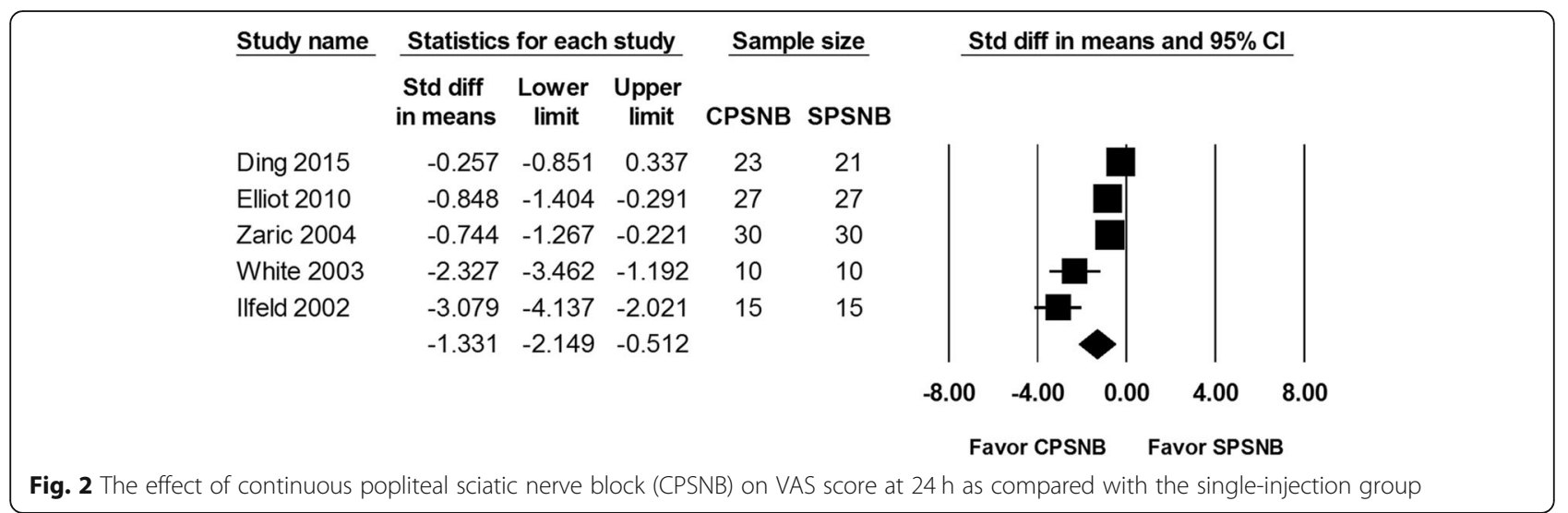

\section{Patient satisfaction scale}

There were 4 studies that recorded the patient satisfaction scale and included 154 patients. However, we are not able to perform analysis of this outcome domain because patient satisfaction scales used in the 4 studies were inconsistent. Two studies showed a higher degree of satisfaction in the CPSNB group compared with the single-injection group [7,9], while the other two studies did not find a significant difference $[10,11]$.

\section{Postoperative admission}

The postoperative admission rate was reported in 3 studies and a total of 134 patients were evaluated. No significant difference was found between the CPSNB and the single-injection group. (OR: $-0.260 ; 95 \%$ CI -0.601 to 0.081 ; Heterogeneity: $\mathrm{I}^{2}=0$; Fig. 6). However, the enrolled number was less than the required information size. Therefore, this result was inconclusive (Additional file 5: Figure S5).

\section{Adverse events and complications}

The adverse events and complications of the enrolled studies were extracted and listed in Table 2. There were no major complications associated with the nerve block procedure or with infusion system, including neuropathic symptoms, infection, adverse drug reaction or accidental fall. However, there were some minor complications, including drug leakage $(N=26$ of 187 , $13.9 \%)$, catheter dislodge $(N=9$ of $187,4.8 \%)$, pump malfunction $(N=4$ of $187,2.1 \%)$ and catheter blockade $(N=2$ of $187,1.0 \%)$.

\section{Publication bias}

The results of the risk of bias evaluation for each study is summarized in Fig. 7 and Fig. 8. The allocation concealment bias (selection bias) was regarded as low risk. The completeness of the reported data (reporting bias) was unclear in 3 of the 5 (60\%) studies included in this analysis. The Egger's test revealed no significant publication bias regarding the overall SMD and odds ratio for included outcome parameters. The funnel plots for SMD and log odds ratio of all of the outcome parameters from each study are shown in Additional file 6: Figure S6, Additional file 7: Figure S7, Additional file 8: Figure S8, Additional file 9: Figure S9, Additional file 10: Figure S10.

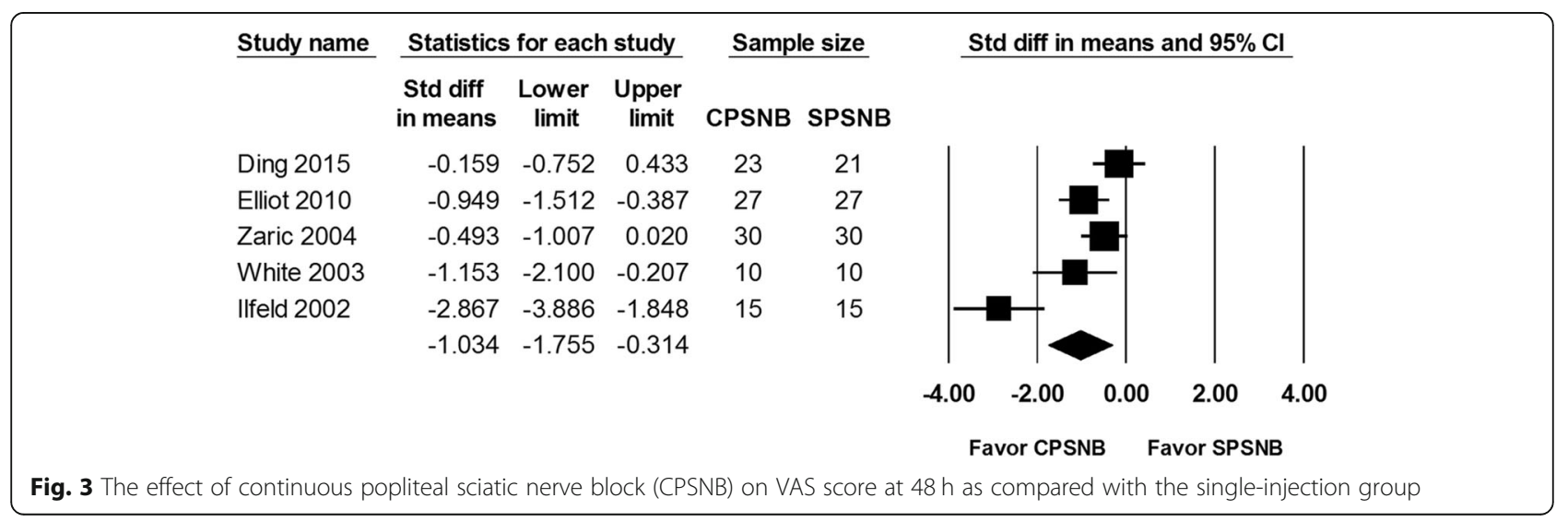




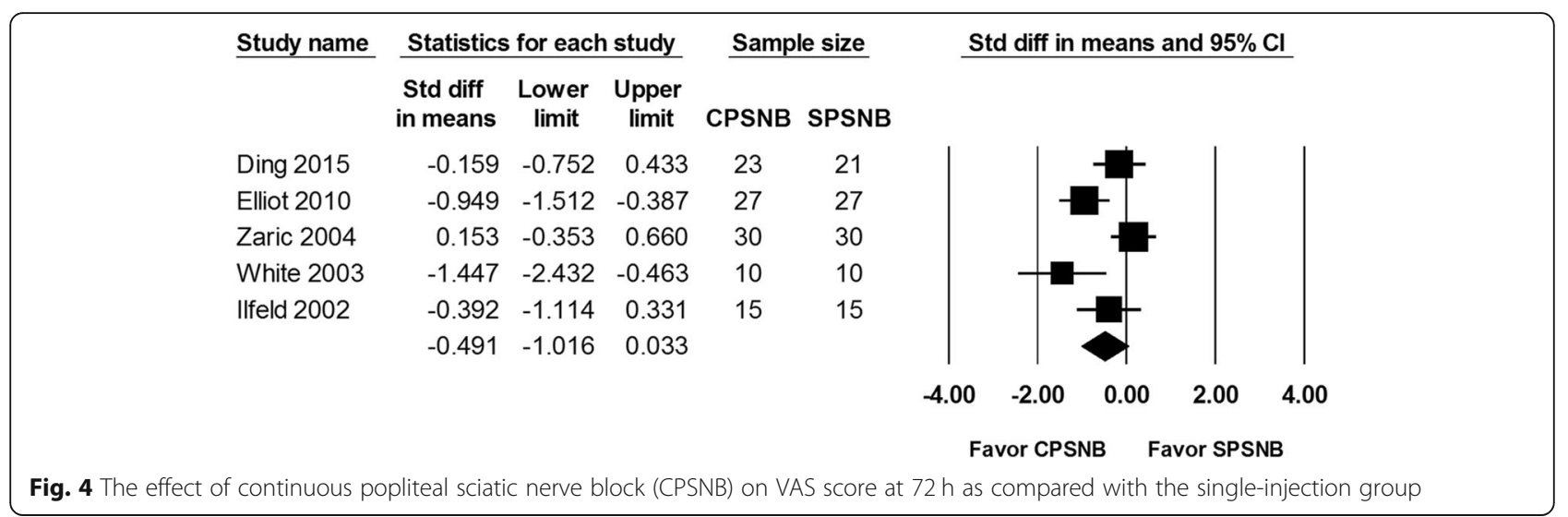

\section{Sensitivity analyses}

A similar result was obtained by using the random-effect model. The SMD and OR were analyzed after excluding one study which contained the smallest sample size. The analysis showed consistent results of outcome parameters in the CPSNB group in comparison with the singleinjection group including VAS score at 24h (SMD: $-1.121 ; 95 \%$ CI -1.969 to -0.274$)$, VAS score at $48 \mathrm{~h}$ (SMD: -1.022 ; $95 \%$ CI -1.877 to -0.167$)$, VAS score at $72 \mathrm{~h}$ (SMD: $-0.327 ; 95 \% \mathrm{CI}-0.823$ to 0.168 ), total amount of oral analgesics consumed at $72 \mathrm{~h}$ (SMD: -0.554 ; $95 \% \mathrm{CI}-1.092$ to -0.276$)$ and postoperative admission (OR: -0.172 ; $95 \%$ CI -0.540 to 0.196 ).

\section{Discussion}

In this meta-analysis, we focused on the efficacy of CPSNB compared with that of single-injection popliteal sciatic nerve block in outpatient foot and ankle surgery. We included 5 RCTs with 208 patients. In comparison with the single-injection nerve block group, patients who received CPSNB presented with less pain (VAS score at 24 and 48 h). Outcome domains including amount of oral analgesics consumed at $72 \mathrm{~h}$, VAS score at $72 \mathrm{~h}$, rate of postoperative admission were considered inconclusive according to the results of trial sequence analysis. There were no major complications including neuropathic symptoms and infection reported in these 5 studies. However, there was a substantial rate of catheter or pump associated complications including catheter dislodge, leakage, irritation, block and pump malfunction.

Effective pain management using regional nerve block as an analgesic adjuvant to anesthesia allows surgeons to perform foot and ankle surgeries as outpatient procedures. Its potential benefits include improved pain control, better patient satisfaction, reduced postoperative opioid consumption and lower costs [21-23]. In the setting of an outpatient procedure, efforts have to be made to prevent unanticipated admissions. In a prospective study of patients who had undergone various ambulatory surgeries, pain was accounted for $12 \%$ of unplanned admissions in which $57.6 \%$ of them were orthopedic patients [4]. The analgesic effects of a single-injection nerve blockade is limited to $15-22 \mathrm{~h}$ [6], which might not be adequate to relief severe pain following foot and ankle surgery up to postoperative day 3 [5]. Therefore, patients might benefit from continuous nerve block to have sustained pain relief.

This study was the first meta-analysis to validate efficacy and safety of CPSNB after foot and ankle surgery as

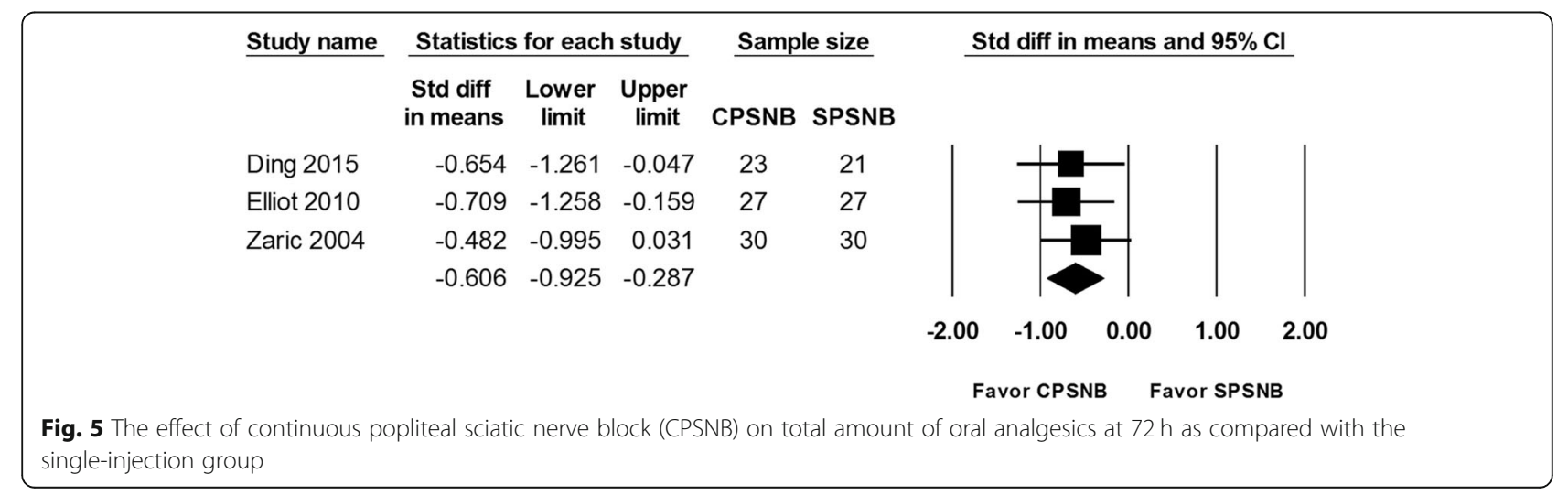




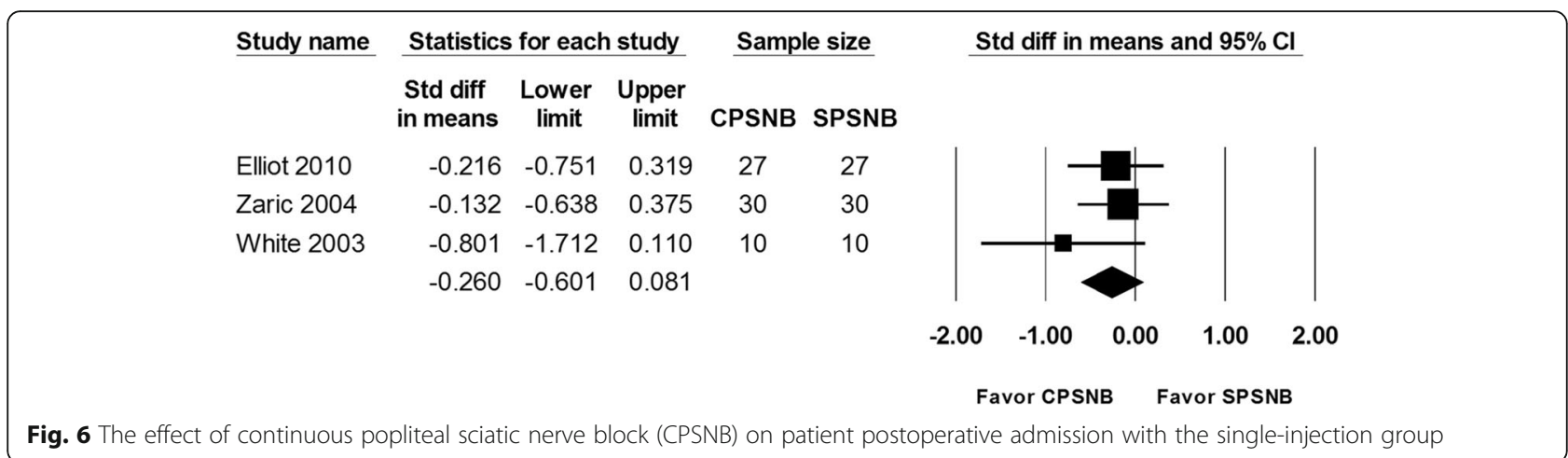

an outpatient procedure, which included five randomized controlled trials for the analysis [7-11]. Compared with the single-injection group, CPSNB was associated with better pain relief up to $48 \mathrm{~h}$. The duration of better pain relief was compatible with the duration of drug administration. In these five studies, a catheter was placed for 48 to $72 \mathrm{~h}$ after surgery [7-11]. Difference of VAS score between the continuous and single-injection popliteal sciatic nerve block group might also be diminished as the post-surgical pain decreases with time. In addition, Sutton et al. and Landorf et al. have previously determined the minimal clinical important difference (MCID) of foot and ankle surgeries. The authors determined that a difference in VAS score of 0.8 can be considered the MCID $[24,25]$. In our study, the difference between two groups in VAS score at $24 \mathrm{~h}$ and $48 \mathrm{~h}$ were 2.28 points and 1.18 , respectively. This further confirms that CPSNB resulted in improved pain management in these patients.

Despite the benefits of better pain control and less analgesics consumption, several inherent risks have to be assessed with popliteal sciatic nerve block and the continuous perineural local anesthetic infusion system. Neuropathic symptoms is one of the major complication associated with popliteal sciatic nerve block that raise the concerns [12-14]. Compére et al. prospectively enrolled 400 patients who received CPSNB. Three major complications were reported, including two neuropathies (0.5\%) [13]. Meanwhile, the incidence of neuropathic symptoms after popliteal sciatic nerve block were higher in recent studies [16-18]. Park et al. conducted a retrospective study of 827 patients evaluating the safety of popliteal sciatic nerve block. Twenty-two (2.7\%) developed neuropathic symptoms secondary to the nerve block and $7(0.8 \%)$ patients had unresolved symptoms at the final follow-up visit [17]. The concern of neuropathic symptoms after popliteal block was supported by the results from Anderson et al., a retrospective study of 1014 patients who received single-injection or continuously popliteal sciatic nerve block. One hundred and thirtyfive (13.3\%) patients developed varying neuropathic symptoms, while most of them $(N=119,87.4 \%)$ reported exclusively sensory deficits. Of the 99 patients who received a continuous infusion with a catheter, 17 (17.1\%) patients developed neuropathic symptoms and 7 (7.0\%) of them were likely related to the popliteal block. These neuropathic symptoms required an average of 58.6 days to resolve, while one of them remained unresolved up to 8 months [18]. In a prospective cohort study conducted by Gartke et al., the incidence of neuropathic symptoms

Table 2 Descriptions of complications in the included studies

\begin{tabular}{|c|c|c|c|}
\hline Author & study design & G1(CPSNB) / G2(SPSNB) & Complications \\
\hline Ding 2015 & $\mathrm{RCT}$ & $23 / 21$ & G1: 5/23 catheter dislodge; 2/23 pump malfunction \\
\hline Elliot 2010 & $\mathrm{RCT}$ & $27 / 27$ & $\begin{array}{l}\text { All: } 6 / 54 \text { drug leakage, } 2 / 54 \text { catheter blockade, } 1 / 54 \text { catheter } \\
\text { dislodge, } 1 / 54 \text { pump malfunction } \\
\text { G1:0/27 adverse drug reaction } \\
\text { G2:1/27 numbness on foot dorsum for } 5 \text { days, recovered }\end{array}$ \\
\hline Zaric 2004 & $\mathrm{RCT}$ & $30 / 30$ & $\begin{array}{l}\text { All: } 5 / 60 \text { drug leakage, } 5 / 60 \text { difficult adaptation to the device, } \\
\text { 1/60 catheter dislodge, } \\
\text { G1: } 0 / 30 \text { adverse drug reaction }\end{array}$ \\
\hline White 2003 & $\mathrm{RCT}$ & $10 / 10$ & $\begin{array}{l}\text { G1:8/10 "tingling sensation" in the foot } \\
\text { G2:1/10 "tingling sensation" in the foot }\end{array}$ \\
\hline Ilfeld 2002 & $\mathrm{RCT}$ & $15 / 15$ & $\begin{array}{l}\text { All: } 15 / 30 \text { drug leakage, } 9 / 30 \text { nonscheduled contact with the } \\
\text { physician, } 2 / 30 \text { catheter dislodge, } 1 / 30 \text { pump malfunction }\end{array}$ \\
\hline
\end{tabular}




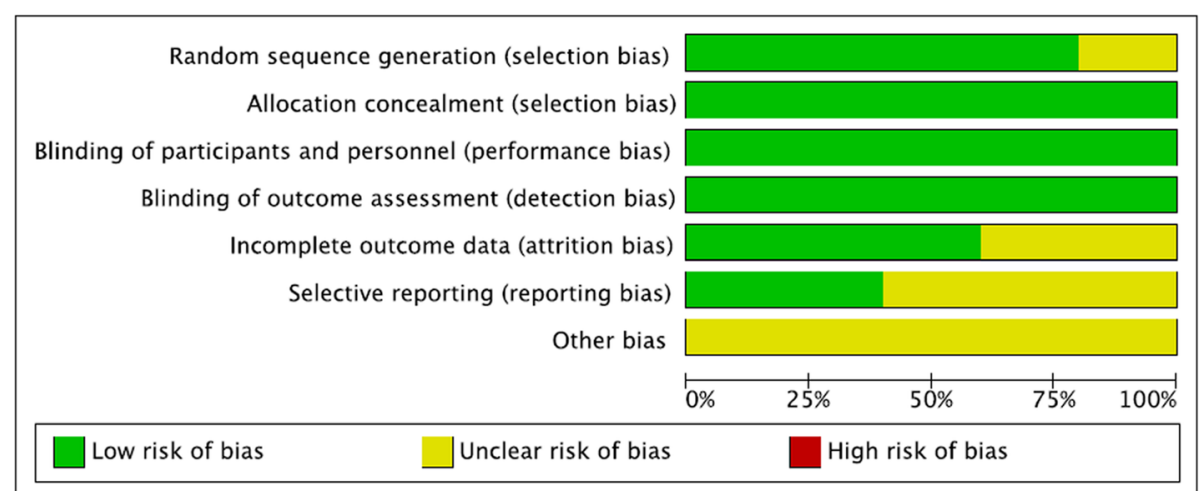

Fig. 7 Summary of the assessment of the risk of bias

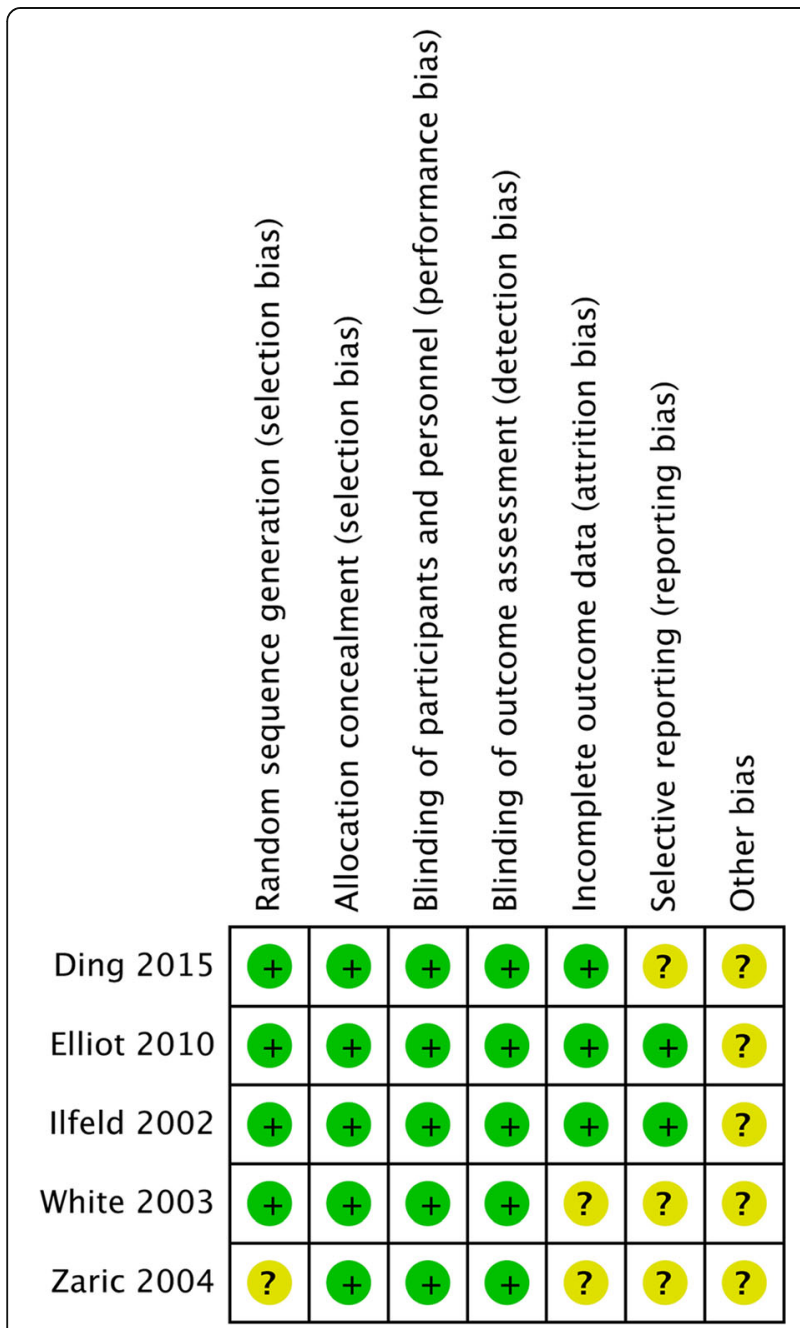

Fig. 8 Results of risk of bias evaluation for each study according to the recommendations of the Cochrane Collaboration after the CPSNB was even higher as $41 \%$ of patients at postoperative 2 weeks and $24 \%$ at 34 weeks had persisted symptoms [16]. Most of the neuropathic symptoms described were sensory deficits of various degrees. The varied incidence of neuropathic symptoms might be due to different study designs (prospective or retrospective data collection), the definition of neuropathic symptoms, and the frequency and duration of follow-up visits [13, 16-18].

Regarding risk factors for neuropathic symptoms, Anderson et al. did not find an association between neuropathic symptoms with smoking, diabetes, tourniquet location or time, use of steroids and/or epinephrine, single or continuous block, and use of ultrasound or nerve stimulator. There was a younger average age in patients who had neuropathic symptoms $(47.3 \pm 15$ years vs. $50.2 \pm 17.2$ years, $P=0.039$ ), compared with those who did not. A higher average tourniquet pressure was found in patients who had neuropathic symptoms $(309.4 \pm 28.9 \mathrm{mmHg}$ vs. $303.4 \pm 24.9 \mathrm{mmHg}, P=0.013)$. Although a statistical significance was noted, the authors suggested that this difference might have minimal clinical significance. Gartke et al. found that smoking history was a borderline significant factor for developing neuropathic symptoms (adjusted OR: 2.25, 95\% CI: 0.96-5.33). The authors did not find tourniquet time, tourniquet application site, experience of the specialist performing the block, use of prophylactic antibiotics, and type of anesthesia to be associated with neuropathic symptoms. Although the exact mechanism causing these neuropathic symptoms remain controversial, some authors have hypothesized that some anesthetics may be directly toxic to nerve fibers [26-28]. Local anesthetics including ropivacaine and bupivacaine block impulse conduction in nerve fibers through inhibition of both sodium ion and potassium ion channels [29]. In addition, the concentration and duration of local anesthetics have been shown to contribute to chemical neurotoxicity in an animal model [27]. Increased amount of anesthetics was 
associated with an elevated intrafascicular pressure that exceeds the nerve capillary perfusion pressure, which would lead to ischemic injury. On the other hand, drugs administered along with the anesthetics such as epinephrine can have a local vasoconstrictive effect that potentially aggravates the ischemic injury [16, 18, 26, 28]. Despite these hypothesis, there are no clinical studies to validate the relationship between the use of common local anesthetics or epinephrine and postoperative neuropathic symptoms. Therefore, studies with high level of evidence are warranted to validate the safety of corticosteroids, epinephrine and each anesthetic. For patients with a smoking history or pre-existing neuropathies, an alternative pain control method to CPSNB should be considered to avoid postoperative neuropathic symptoms.

In the 5 RCTs included in this meta-analysis, Elliot et al. reported a patient in the placebo group who experienced numbness on the dorsal foot which resolved after 5 days [8]. White et al. found a higher incidence of "tingling sensation" on the foot in the CPSNB group ( $80 \%$ versus $10 \%$ in the placebo group) [10]. The duration of this sensation was not described. Anderson et al. defined abnormal postoperative neuropathic symptoms as burning, pain, numbness, or tingling in the operative limb that persisted for at least 7 days following a single injection or after catheter removal for continuous infusions. According to this definition, there were no neuropathic events in the 5 studies included in this meta-analysis.

Although no major complications were mentioned in either group, there was a significantly higher rate of minor complications associated with the infusion system, including drug leakage, catheter dislodge, pump malfunction and catheter blockade. When using a portable infusion system, patients have to take an extra responsibility that comes with the device. Those minor complications might lead to a significant rate of nonscheduled contact with the physician $(N=9$ of $30,30 \%)$ [11] and might affect the overall satisfaction rate. A prudent assessment of patients' desire and a comprehensive verbal and written instruction would be warranted.

There are several limitations that should be recognized. First, the small sample size and a high heterogeneity of clinical settings between studies, including age, gender, doses and regimens used in CPSNB, surgical approaches, catheter locations and types of anesthesia may effect the results, which should be interpreted with caution. Second, we searched only for English articles but not articles in other languages or unpublished data. This might be a potential source for publication bias. Third, there was an inherent flawed setting in these studies that compared CPSNB with the placebo group. A potential bias associated with a "wash out" effect existed in the placebo group. Continuous saline infusion in the placebo group might have diluted the initial, single-injection regional block and shortened the duration. This can lead to a bias toward the efficacy of CPSNB [11]. Finally, several important outcome parameters with regard to safety concerns of CPSNB including neuropathic symptoms and infection could not be evaluated in our metaanalysis because 4 of 5 RCTs enrolled in this metaanalysis have placed a catheter with saline infusion in the single-injection group to enable blinding of patients. Thus we are unable to present comparative results of major and minor complications between CPSNB and single-injection group but rather an overall descriptive data. Therefore, future studies can be designed to place an emphasis in determining the incidence difference of complications between CPSNB and single-injection to provide a more comprehensive result.

\section{Conclusion}

This meta-analysis revealed that continuous popliteal sciatic nerve block can lead to better pain relief for patients who had undergone foot and ankle surgery. Both methods appear to be safe as none of the patients experienced neuropathic symptoms or infection. Further studies with larger sample size are needed to compare the risk of major complications between the two methods.

\section{Supplementary information}

Supplementary information accompanies this paper at https://doi.org/10. 1186/s12891-019-2822-7.

\footnotetext{
Additional file 1: Figure S1. Trial sequential analysis for the effect of continuous popliteal sciatic nerve block (CPSNB) on VAS score at $24 \mathrm{~h}$ as compared with the single-injection group. The lower half of the graph below the 0 axis represents the area of VAS score at $24 \mathrm{~h}$ in SPSNB, and the upper half represents the VAS score at $24 \mathrm{~h}$ in CPSNB. Solid lines (Brown) at +1.96 and -1.96 on $Y$ axis represent the conventional model boundaries for TSA with an a of $5 \%$. The required information size (IS) for the conventional boundary model is 50 (shown on X-axis). The red lines represent the a-spending boundary (upper O'Brien-Fleming with a of 5\% and $\beta$ of $20 \%)$. The area to the left of the wedge formed by the red lines of a-spending is the area of futility. The area to the right of the wedge is the area of equivalence. The RIS for a-spending boundary model is 66 (shown on vertical line intersecting $X$-axis in red) Cumulative $Z$ scores fall within the superior range for both conventional boundary and the aspending boundary of the O'Brien-Fleming model.

Additional file 2: Figure S2. Trial sequential analysis for the effect of continuous popliteal sciatic nerve block (CPSNB) on VAS score at $48 \mathrm{~h}$ as compared with the single-injection group. The lower half of the graph below the 0 axis represents the area of VAS score at $48 \mathrm{~h}$ in SPSNB, and the upper half represents the VAS score at $48 \mathrm{~h}$ in CPSNB. Solid lines (Brown) at +1.96 and -1.96 on $Y$ axis represent the conventional model boundaries for TSA with an a of 5\%. The required information size (IS) for the conventional boundary model is 50 (shown on X-axis). The red lines represent the a-spending boundary (upper O'Brien-Fleming with a of 5\% and $\beta$ of $20 \%$ ). The area to the left of the wedge formed by the red lines of a-spending is the area of futility. The area to the right of the wedge is the area of equivalence. The RIS for a-spending boundary model is 76 (shown on vertical line intersecting $X$-axis in red) Cumulative $Z$ scores fall within the superior range for both conventional boundary and the $a-$ spending boundary of the O'Brien-Fleming model.
} 
Additional file 3: Figure S3. Trial sequential analysis for the effect of continuous popliteal sciatic nerve block (CPSNB) on VAS score at $72 \mathrm{~h}$ as compared with the single-injection group. The lower half of the graph below the 0 axis represents the area of VAS score at $72 \mathrm{~h}$ in SPSNB, and the upper half represents the VAS score at $72 \mathrm{~h}$ in CPSNB. Solid lines (Brown) at +1.96 and -1.96 on $Y$ axis represent the conventional model boundaries for TSA with an a of 5\%. The required information size (IS) for the conventional boundary model is 208 (shown on X-axis). The red lines represent the a-spending boundary (upper O'Brien-Fleming with a of $5 \%$ and $\beta$ of 20\%). The area to the left of the wedge formed by the red lines of a-spending is the area of futility. The area to the right of the wedge is the area of equivalence. The RIS for a-spending boundary model is 241 (shown on vertical line intersecting $X$-axis in red). Cumulative $Z$ score didn't reach the boundary of RIS which implicated inconclusive result.

Additional file 4: Figure S4. Trial sequential analysis for the effect of continuous popliteal sciatic nerve block (CPSNB) on oral analgesics consumption at $72 \mathrm{~h}$ as compared with the single-injection group. The lower half of the graph below the 0 axis represents the area of oral analgesics consumption at $72 \mathrm{~h}$ in SPSNB, and the upper half represents the oral analgesics consumption at $72 \mathrm{~h}$ in CPSNB. Solid lines (Brown) at + 1.96 and -1.96 on $Y$ axis represent the conventional model boundaries for TSA with an a of 5\%. The required information size (IS) for the conventional boundary model is 158 (shown on X-axis). The red lines represent the a-spending boundary (upper O'Brien-Fleming with a of 5\% and $\beta$ of 20\%). The area to the left of the wedge formed by the red lines of a-spending is the area of futility. The area to the right of the wedge is the area of equivalence. The RIS for a-spending boundary model is 649 (shown on vertical line intersecting $X$-axis in red). Cumulative $Z$ score didn't reach the boundary of RIS which implicated inconclusive result.

Additional file 5: Figure S5. Trial sequential analysis for the effect of postoperative admission as compared with the single-injection group. The lower half of the graph below the 0 axis represents the area of oral analgesics consumption at $72 \mathrm{~h}$ in SPSNB, and the upper half represents postoperative admission in CPSNB. Solid lines (Brown) at +1.96 and -

1.96 on $Y$ axis represent the conventional model boundaries for TSA with an a of $5 \%$. The required information size (IS) for the conventional boundary model is 208 (shown on $\mathrm{X}$-axis). The red lines represent the $\mathrm{a}$ spending boundary (upper O'Brien-Fleming with a of $5 \%$ and $\beta$ of $20 \%$ ). The area to the left of the wedge formed by the red lines of $a$-spending is the area of futility. The area to the right of the wedge is the area of equivalence. The RIS for a-spending boundary model is 980 (shown on vertical line intersecting $X$-axis in red). Cumulative $Z$ score didn't reach the boundary of RIS which implicated inconclusive result.

Additional file 6: Figure S6. Funnel plot of VAS score at $24 \mathrm{~h}$.

Additional file 7: Figure S7. Funnel plot of VAS score at $48 \mathrm{~h}$.

Additional file 8: Figure S8. Funnel plot of VAS score at $72 \mathrm{~h}$.

Additional file 9: Figure S9. Funnel plot of total amount of oral analgesics at $72 \mathrm{~h}$.

Additional file 10: Figure S10. Funnel plot of patient postoperative admission.

\section{Abbreviations}

CPSNB: Continuous popliteal sciatic nerve block; OR: Odds ratio; PACU: Postanesthesia care unit; PRISMA: Preferred reporting items for systematic reviews and meta-analysis; RCT: Randomized controlled trial; SMD: Standardized mean difference; VAS: Visual analog scale

\section{Acknowledgements}

Not applicable.

\section{Authors' contributions}

Concept, literature search and data collection: H-HM, S-WT. Statistics, data analysis and interpretation: H-HM, S-WT, P-KW, W-MC. Drafting article: H-HM, T-FAC, S-WT, C-FC. Critical revision of article: T-FAC, S-WT, C-FC, P-KW, W-MC. All authors have read and approved the final manuscript.

\section{Funding}

This study received no funding.
Availability of data and materials

The information to access the data used in the study, is included within this article.

Ethics approval and consent to participate

Not applicable.

\section{Consent for publication}

Not applicable.

\section{Competing interests}

The authors declare that they have no competing interests.

Received: 30 April 2019 Accepted: 10 September 2019

Published online: 10 October 2019

\section{References}

1. Iffeld BM, Enneking FK. Continuous peripheral nerve blocks at home: a review. Anesth Analg. 2005;100(6):1822-33.

2. Iffeld BM, Esener DE, Morey TE, Enneking FK. Ambulatory perineural infusion: the patients' perspective. Reg Anesth Pain Med. 2003;28(5):418-23.

3. Wu CL, Berenholtz SM, Pronovost PJ, Fleisher LA. Systematic review and analysis of postdischarge symptoms after outpatient surgery. Anesthesiology. 2002;96(4):994-1003.

4. Fortier J, Chung F, Su J. Unanticipated admission after ambulatory surgery-a prospective study. Can J Anaesth. 1998;45(7):612-9.

5. Chou LB, Wagner D, Witten DM, Martinez-Diaz GJ, Brook NS, Toussaint M, Carroll IR. Postoperative pain following foot and ankle surgery: a prospective study. Foot Ankle Int. 2008;29(11):1063-8.

6. Rasmussen SB, Saied NN, Bowens C Jr, Mercaldo ND, Schildcrout JS, Malchow RJ. Duration of upper and lower extremity peripheral nerve blockade is prolonged with dexamethasone when added to ropivacaine: a retrospective database analysis. Pain Med. 2013;14(8):1239-47.

7. Ding DY, Manoli A 3rd, Galos DK, Jain S, Tejwani NC. Continuous popliteal sciatic nerve block versus single injection nerve block for ankle fracture surgery: a prospective randomized comparative trial. J Orthop Trauma. 2015; 29(9):393-8

8. Elliot R, Pearce CJ, Seifert C, Calder JD. Continuous infusion versus single bolus popliteal block following major ankle and hindfoot surgery: a prospective, randomized trial. Foot Ankle Int. 2010;31(12):1043-7.

9. Zaric D, Boysen K, Christiansen J, Haastrup U, Kofoed H, Rawal N. Continuous popliteal sciatic nerve block for outpatient foot surgery--a randomized, controlled trial. Acta Anaesthesiol Scand. 2004:48(3):337-41.

10. White PF, Issioui T, Skrivanek GD, Early JS, Wakefield C. The use of a continuous popliteal sciatic nerve block after surgery involving the foot and ankle: does it improve the quality of recovery? Anesth Analg. 2003; 97(5):1303-9.

11. Iffeld BM, Morey TE, Wang RD, Enneking FK. Continuous popliteal sciatic nerve block for postoperative pain control at home: a randomized, doubleblinded, placebo-controlled study. Anesthesiology. 2002;97(4):959-65.

12. Compere V, Cornet C, Fourdrinier V, Maitre AM, Mazirt N, Biga N, Dureuil B. Thigh abscess as a complication of continuous popliteal sciatic nerve block. $\mathrm{Br} J$ Anaesth. 2005;95(2):255-6.

13. Compere V, Rey N, Baert O, Ouennich A, Fourdrinier V, Roussignol X, Beccari R, Dureuil B. Major complications after 400 continuous popliteal sciatic nerve blocks for post-operative analgesia. Acta Anaesthesiol Scand. 2009; 53(3):339-45.

14. Dott D, Canlas C, Sobey C, Obremskey W, Thomson AB. Necrotizing fasciitis as a complication of a continuous sciatic nerve catheter using the lateral popliteal approach. Reg Anesth Pain Med. 2016;41(6):728-30.

15. Hajek V, Dussart C, Klack F, Lamy A, Martinez JY, Laine P, Mazurier L, Guilloton L, Drouet A. Neuropathic complications after 157 procedures of continuous popliteal nerve block for hallux valgus surgery. A retrospective study. Orthop Traumatol Surg Res. 2012;98(3):327-33.

16. Gartke K, Portner O, Taljaard M. Neuropathic symptoms following continuous popliteal block after foot and ankle surgery. Foot Ankle Int. 2012:33(4):267-74

17. Park YU, Cho JH, Lee DH, Choi WS, Lee HD, Kim KS. Complications after multiple-site peripheral nerve blocks for foot and ankle surgery compared with popliteal sciatic nerve block alone. Foot Ankle Int. 2018;39(6):731-5. 
18. Anderson JG, Bohay DR, Maskill JD, Gadkari KP, Hearty TM, Braaksma W, Padley MA, Weaver KT. Complications after popliteal block for foot and ankle surgery. Foot Ankle Int. 2015;36(10):1138-43.

19. Saporito A, Sturini E, Petri J, Borgeat A, Aguirre JA. Case report: unusual complication during outpatient continuous regional popliteal analgesia. Can J Anaesth. 2012;59(10):958-62.

20. Wetterslev J, Thorlund K, Brok J, Gluud C. Trial sequential analysis may establish when firm evidence is reached in cumulative meta-analysis. I Clin Epidemiol. 2008;61(1):64-75.

21. Gupta A, Kumar K, Roberts MM, Sanders AE, Jones MT, Levine DS, O'Malley MJ, Drakos MC, Elliott AJ, Deland JT, et al. Pain management after outpatient foot and ankle surgery. Foot Ankle Int. 2018;39(2):149-54.

22. Saporito A, Sturini E, Borgeat A, Aguirre J. The effect of continuous popliteal sciatic nerve block on unplanned postoperative visits and readmissions after foot surgery--a randomised, controlled study comparing day-care and inpatient management. Anaesthesia. 2014;69(11):1197-205.

23. Saporito A, Calciolari S, Ortiz LG, Anselmi L, Borgeat A, Aguirre J. A cost analysis of orthopedic foot surgery: can outpatient continuous regional analgesia provide the same standard of care for postoperative pain control at home without shifting costs? Eur J Health Econ. 2016;17(8):951-61.

24. Landorf KB, Radford JA, Hudson S. Minimal important difference (MID) of two commonly used outcome measures for foot problems. Journal of foot and ankle research. 2010;3:7.

25. Sutton RM, McDonald EL, Shakked RJ, Fuchs D, Raikin SM. Determination of minimum clinically important difference (MCID) in visual analog scale (VAS) pain and foot and ankle ability measure (FAAM) scores after hallux Valgus surgery. Foot Ankle Int. 2019;40(6):687-93.

26. Hogan QH. Pathophysiology of peripheral nerve injury during regional anesthesia. Reg Anesth Pain Med. 2008;33(5):435-41.

27. Myers RR, Kalichman MW, Reisner LS, Powell HC. Neurotoxicity of local anesthetics: altered perineurial permeability, edema, and nerve fiber injury. Anesthesiology. 1986;64(1):29-35.

28. Sorenson EJ. Neurological injuries associated with regional anesthesia. Reg Anesth Pain Med. 2008;33(5):442-8.

29. Kuthiala G, Chaudhary G. Ropivacaine: a review of its pharmacology and clinical use. Indian J Anaesth. 2011;55(2):104-10.

\section{Publisher's Note}

Springer Nature remains neutral with regard to jurisdictional claims in published maps and institutional affiliations.

Ready to submit your research? Choose BMC and benefit from:

- fast, convenient online submission

- thorough peer review by experienced researchers in your field

- rapid publication on acceptance

- support for research data, including large and complex data types

- gold Open Access which fosters wider collaboration and increased citations

- maximum visibility for your research: over $100 \mathrm{M}$ website views per year

At $\mathrm{BMC}$, research is always in progress.

Learn more biomedcentral.com/submissions 